\title{
About the Torsional Constant for thin-walled rod with open
}

\author{
cross-section \\ Duan Jin ${ }^{1, a}$, Li Yun-gui ${ }^{1}$ \\ ${ }^{1}$ China State Construction Technical Center, Beijing, 101300, PR China \\ a duanjin78@126.com
}

Key words: open thin-walled rod, torsional constant, finite element method

Abstract: This paper presents theoretically the torsional inertia moment for open thin-walled rod, based on the theory of thin-walled shell. In the deduction, the rigid contour hypothesis is adopted and the cross-sectional warp is taken into account. With the above torsional inertia moment obtained and using the similar method for closed beam elements in Reference [1], the stiffness matrix of thin-walled beam element with open cross-section is derived with the warping of cross-section considered. Other than the traditional stiffness matrix of beam element having twelve DOFs, i.e. six for each node, this stiffness matrix is corresponding to fourteen DOFs, i.e. seven for each node. This matrix could be used for the finite element analysis of open thin-walled rod, with section warping considered.

\section{Introduction}

In building structures, the thin-walled rod with open cross-section is widely used. It is usually analyzed by theoretical solution or finite element method (FEM) [1-3] for simple structure or complicated structure respectively. Either way, there is an important or even critical property of the cross-section needing to be determined first. It is the torsional inertia moment for open thin-walled cross-section.

In this paper, the torsional inertia moment for open thin-walled rod is derived theoretically based on the theory of thin-walled shell. In the deduction, the rigid contour hypothesis is adopted and the cross-sectional warp is taken into account. With the above torsional inertia moment obtained and using the similar method for closed beam elements in Reference [1], the stiffness matrix of thin-walled beam element with open cross-section is derived with the warping of cross-section considered. Other than the traditional stiffness matrix of beam element having twelve DOFs, i.e. six for each node, this stiffness matrix is corresponding to fourteen DOFs, i.e. seven for each node. This matrix could be used for the finite element analysis of open thin-walled rod, with section warping considered.

\section{The torsional constant for thin-walled rod with open cross-section}

Figure 1 gives an illustration of the cross section of an open thin-walled rod. There are three sets of coordinate system, i.e. Cartesian coordinate system located at the centroid of cross-section, contour coordinate system whose original location is moving along the midcourt line of the thin-walled cross section, and coordinate system located at the shear center, whose axes are parallel to that of contour coordinate system. Detailedly speaking, select arbitrary point $P$ at the midcourt line $C$ as the original point, and denote the vectors 
along the wall thickness orientation and the tangential midcourt line as $n$ and $s$ respectively, then the contour coordinate system is obtained. Offset the original point of contour coordinate system to the shear point, and denote the axes parallel to $n$ and $s$ as $q$ and $\xi$ respectively, then another coordinate system is obtained.

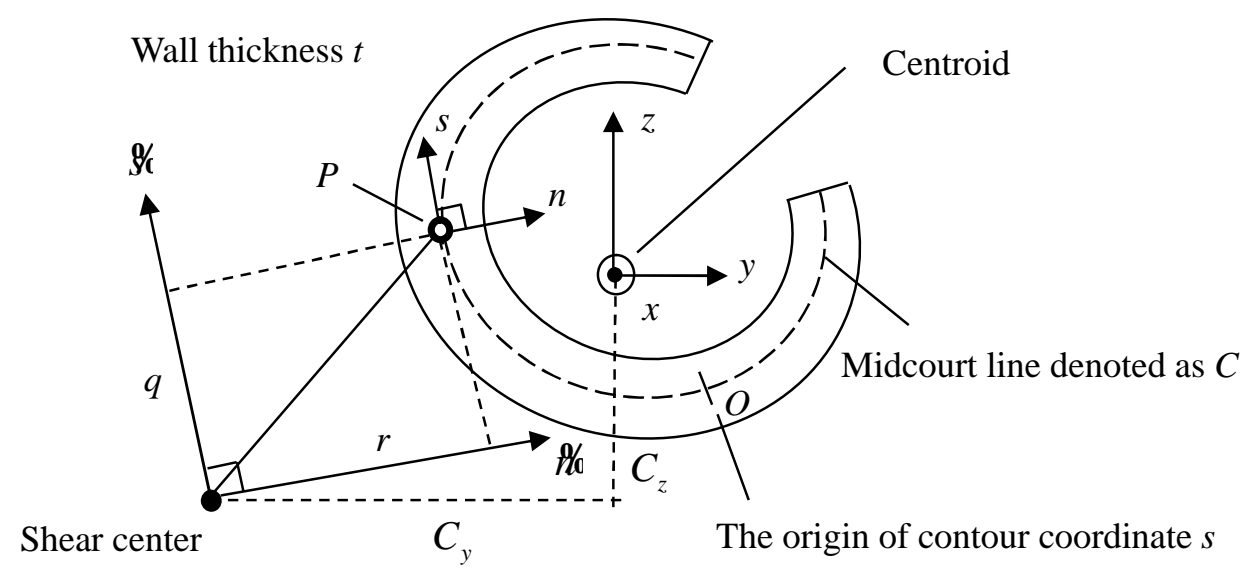

Figure 1 Cross section of an open thin-walled rod

According to Reference [5], the warping function of the cross-section is as following:

$$
\omega(n, s)=\bar{\omega}+\overline{\bar{\omega}}
$$

Where $\bar{\omega}$ denotes the contour warping function, equaling the sectorial area of the midcourt line, $\overline{\bar{\omega}}$ denotes the thickness warping function, see the following Equation (2):

$$
\bar{\omega}=\int_{0}^{s} r_{c}(s) d s+\overline{\omega_{0}}, \quad \overline{\bar{\omega}}=-n q_{c}(s)
$$

Where $\bar{\omega}_{0}$ denotes the contour warping function at $\mathrm{s}=0 ; r_{c}, q_{c}$ denotes $r$ and $q$ respectively corresponding to the midcourt line

The following Equation (3) could be obtained from Equation (1) and (2):

$$
\frac{\partial \omega}{\partial s}=r_{c}(s)-n, \quad \frac{\partial \omega}{\partial n}=-q_{c}(s)
$$

The following equations could be obtained for pure torsional problem at cylindrical coordinate system xns:

$$
u_{x}=-\omega(n, s) \chi, \quad u_{n}=-q(s) \theta_{x}, \quad u_{s}=r(s) \theta_{x}
$$

The shearing strain is as follow:

$$
\left\{\begin{array}{l}
\gamma_{x n}=\frac{\partial u_{x}}{\partial n}+\frac{\partial u_{n}}{\partial x}=\left(-q_{c}+q\right) \theta_{x, x} \\
\gamma_{x s}=\frac{\partial u_{x}}{\partial s}+\frac{\partial u_{s}}{\partial x}=\left(r(s)-\left(r_{c}(s)-n\right)\right) \theta_{x, x}
\end{array}\right.
$$

Observing Figure 1, the following equation could be obtained: 


$$
r(s)=r_{c}(s)+n, \quad q(s)=q_{c}(s)
$$

Substitute Equation (6) into (5), the following Equation (7) could be derived:

$$
\gamma_{x n}=0, \quad \gamma_{x s}=2 n \theta_{x, x}
$$

The potential variational formula for thin-walled rod is as follow [1-3]:

$$
\delta \Pi=\int_{l} \int_{A} G \gamma_{x s} \delta \gamma_{x s} d A d l=\int_{l} G J \theta_{x, x} \delta \theta_{x, x} d l
$$

Where $J$ denotes the torsional inertia moment, see the following equation for details:

$$
J=\int_{A}(2 n)^{2} d A=\int_{s_{1}}^{s_{2}} \int_{-t / 2}^{t / 2}(2 n)^{2} d n d s=\int_{s_{1}}^{s_{2}} \frac{1}{3} t^{3} d s
$$

It is obviously that $J$ denotes St.Venant torsional constant.

Deducing the above equations in rectangular coordinate system, then

$$
J^{\prime}=\int_{A}\left[\left(z-C_{z}+\omega_{, y}\right)^{2}+\left(y-C_{y}-\omega_{, z}\right)^{2}\right] d A
$$

Considering the fact that torsional inertia moment is the inherent nature of the cross-section and has nothing to do with the coordinate system, the following equation could be derived:

$$
J=J^{\prime}=\int_{A}\left[\left(z-C_{z}+\omega_{, y}\right)^{2}+\left(y-C_{y}-\omega_{, z}\right)^{2}\right] d A
$$

\section{The stiffness matrix of thin-walled beam element with open cross-section}

Use the similar method for closed beam elements, see Reference [1], together with the above Equation (11), the linear stiffness matrix of open thin-walled beam element could be derived as follow:

$$
\mathbf{K}=\left[\begin{array}{ll}
\mathbf{K}^{11} & \mathbf{K}^{12} \\
\mathbf{K}^{21} & \mathbf{K}^{22}
\end{array}\right]
$$

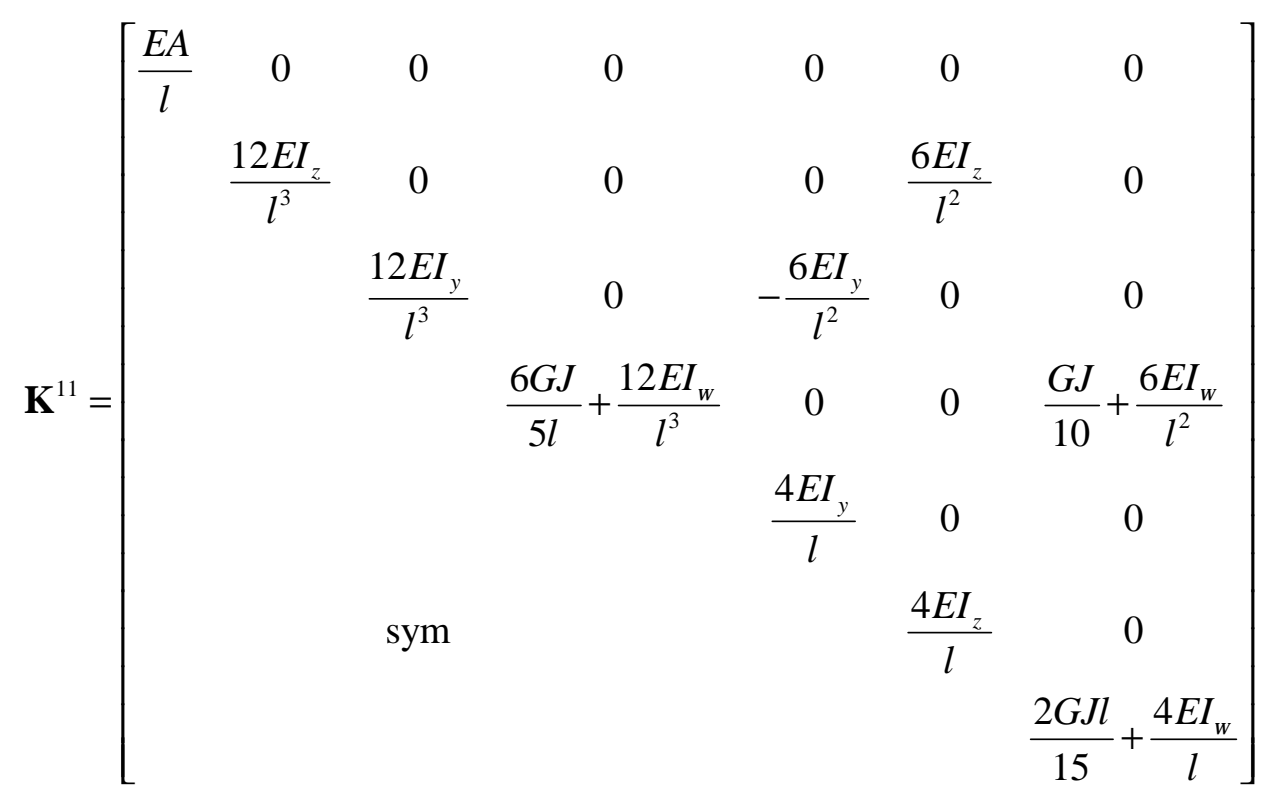


$\mathbf{K}^{12}=\left[\begin{array}{ccccccc}-\frac{E A}{l} & 0 & 0 & 0 & 0 & 0 & 0 \\ 0 & -\frac{12 E I_{z}}{l^{3}} & 0 & 0 & 0 & \frac{6 E I_{z}}{l^{2}} & 0 \\ 0 & 0 & -\frac{12 E I_{y}}{l^{3}} & 0 & -\frac{6 E I_{y}}{l^{2}} & 0 & 0 \\ 0 & 0 & 0 & -\frac{6 G J}{5 l}-\frac{12 E I_{\omega}}{l^{3}} & 0 & 0 & \frac{G J}{10}+\frac{6 E I_{\omega}}{l^{2}} \\ 0 & 0 & \frac{6 E I_{y}}{l^{2}} & 0 & \frac{2 E I_{y}}{l} & 0 & 0 \\ 0 & -\frac{6 E I_{z}}{l^{2}} & 0 & 0 & 0 & \frac{2 E I_{z}}{l} & 0 \\ 0 & 0 & 0 & -\frac{G J}{10}-\frac{6 E I_{\omega}}{l^{2}} & 0 & 0 & -\frac{G J l}{30}+\frac{2 E I_{\omega}}{l}\end{array}\right]$

$\mathbf{K}^{21}=\left[\begin{array}{ccccccc}-\frac{E A}{l} & 0 & 0 & 0 & 0 & 0 & 0 \\ 0 & -\frac{12 E I_{z}}{l^{3}} & 0 & 0 & 0 & -\frac{6 E I_{z}}{l^{2}} & 0 \\ 0 & 0 & -\frac{12 E I_{y}}{l^{3}} & 0 & \frac{6 E I_{y}}{l^{2}} & 0 & 0 \\ 0 & 0 & 0 & -\frac{6 G J}{5 l}-\frac{12 E I_{\omega}}{l^{3}} & 0 & 0 & -\frac{G J}{10}-\frac{6 E I_{\omega}}{l^{2}} \\ 0 & 0 & -\frac{6 E I_{y}}{l^{2}} & 0 & \frac{2 E I_{y}}{l} & 0 & 0 \\ 0 & \frac{6 E I_{z}}{l^{2}} & 0 & 0 & 0 & \frac{2 E I_{z}}{l} & 0 \\ 0 & 0 & 0 & \frac{G J}{10}+\frac{6 E I_{\omega}}{l^{2}} & 0 & 0 & -\frac{G J l}{30}+\frac{2 E I_{\omega}}{l}\end{array}\right]$

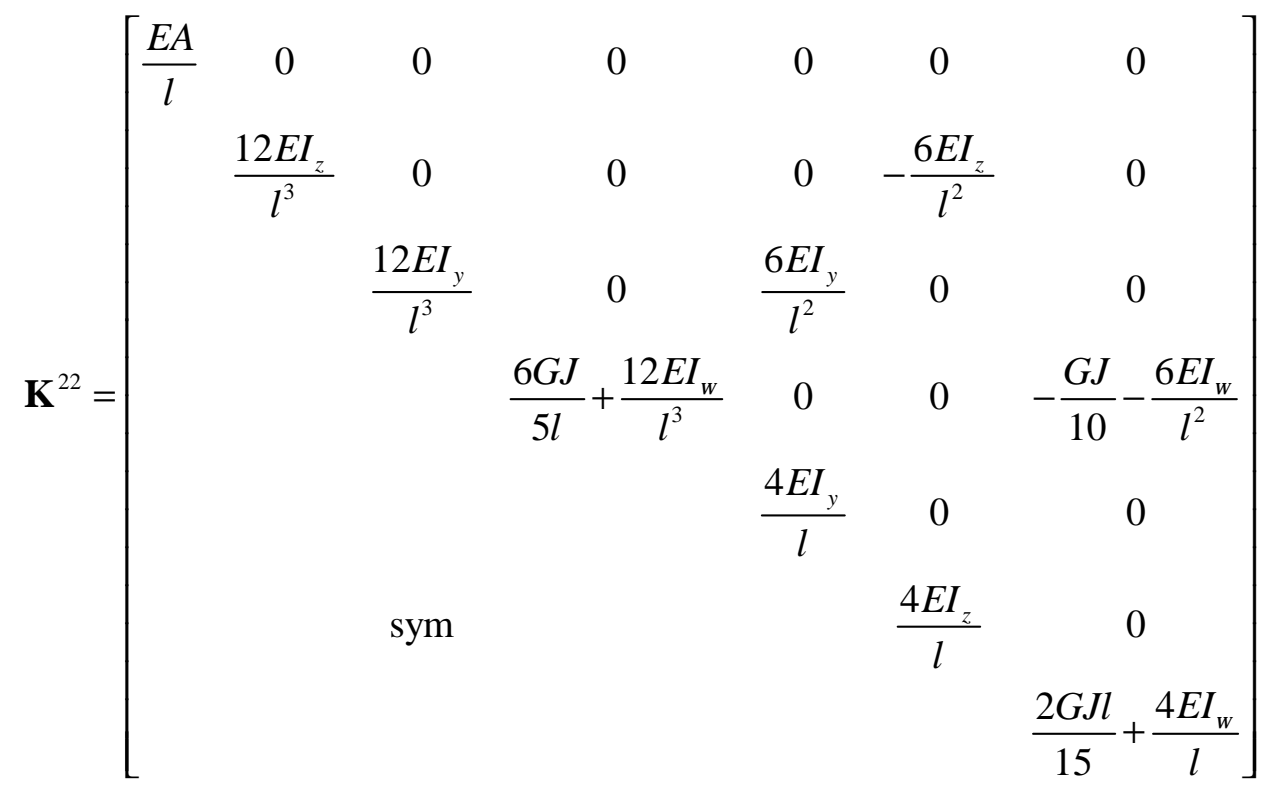

Where $I_{y}$ and $I_{z}$ denotes the principal moment of inertia according to axis $\mathrm{v}$ and $\mathrm{z}$ respectively; 
$J$ denotes the torsional moment of inertia for open thin-walled rod, see Equation (11); $I_{\omega}$ denotes the cross section's warping moment of inertia, see the following equation:

$$
I_{\omega}=\int_{A} \omega^{2} d A
$$

Where $\omega$ denotes the warping function of cross-section $[4,5,6]$

\section{Summary}

In this paper, the torsional inertia moment for open thin-walled rod is derived theoretically based on the theory of thin-walled shell. In the deduction, the rigid contour hypothesis is adopted and the cross-sectional warp is taken into account. With the above torsional inertia moment obtained and using the similar method for closed beam elements in Reference [1], the stiffness matrix of thin-walled beam element with open cross-section is derived with the warping of cross-section considered. Other than the traditional stiffness matrix of beam element having twelve DOFs, i.e. six for each node, this stiffness matrix is corresponding to fourteen DOFs, i.e. seven for each node. This matrix could be used for the finite element analysis of open thin-walled rod, with section warping considered.

\section{Reference}

[1] Bathe K J, Bolourchi S. Large Displacement Analysis of Three-Dimensional Beam Structures. Int. J. Num. Mech. Engng., 1979, 14: 961-986

[2] Bathe K J. Finite Element Procedures. NJ: Prentice-Hall, 1996

[3] Wang X C. Finite Element Method. Beijing, China: Tsinghua University Press, 2003 (in Chinese)

[4] Vlasov V Z. Thin walled elastic beams. Israel Program for Scientific Translation, Jerusalem, 1961

[5] Hu H C \& Xie B M. Statics for Elastic Thin-walled Rod. 1955, Beijing, China: Science Press (in Chinese, Translated from Russian)

[6] Gjelsvik A. The theory of thin walled bars. New York: Wiley, 1981 